\title{
Flow over Rough-Walled Circular Cylinder IN THE CRITICAL AREA
}

\author{
Lenka LAUSOVA ${ }^{1}$, Ivan KOLOS ${ }^{2}$, Vladimira MICHALCOVA ${ }^{3}$ \\ ${ }^{1}$ Department of Structural Mechanics, Faculty of Civil Engineering, VSB - Technical University of Ostrava \\ Ludvika Podeste 1875/17, 70833 Ostrava - Poruba, Czech Republic \\ ${ }^{2}$ Department of Structural Mechanics, Faculty of Civil Engineering, VSB - Technical University of Ostrava \\ Ludvika Podeste 1875/17, 70833 Ostrava - Poruba, Czech Republic \\ ${ }^{3}$ Department of Structural Mechanics, Faculty of Civil Engineering, VSB - Technical University of Ostrava \\ Ludvika Podeste 1875/17, 70833 Ostrava - Poruba, Czech Republic \\ lenka.lausova@vsb.cz, ivan.kolos@vsb.cz, vladimira.michalcova@vsb.cz
}

DOI: $10.31490 /$ tces-2018-0001

\begin{abstract}
The article deals with the flow over a roughwalled circular cylinder in the critical area at high Re numbers. The subject of the paper is a comparison of the standard calculation of the aerodynamic drag coefficient with numerical modeling. Numerical tasks are solved by the simplified geometry of the smooth cylinder, where the influence of the rough surface is given by the equivalent aerodynamic roughness, and also by the model with the real geometry of the rough casing of the cylinder.
\end{abstract}

\section{Keywords}

CFD, SST k-omega, circular cylinder, high Reynolds number, boundary layer, wall function, aerodynamic roughness, near-wall modeling, aerodynamic drag coefficient.

\section{Introduction}

The purpose of this study is to show the approach to determining sheathing roughness of flow over a circular cylinder at high $R e$ numbers by numerical modeling using the finite volume method in ANSYS Fluent software. This is a complex problem of flow in the critical area, the solution of which is dealt with by many international departments, whether in experimental [1, 2, [3, 4] or numerical [5], [6], 17, 8], [9], 10], 11], [12, [13] research.

The article is based on a practical task to determine the effect of the load (aerodynamic drag coefficient $c_{d}$ ) of the flow over a smokestack, the casing of which is formed by a shaped sheet. Calculation of the drag coefficient $c_{d}$ according to the currently valid standard depends on the value of the Reynolds number $(R e)$ and takes into account the roughness of the flow over body. This study follows the previous papers [14, [15]. Drag coefficient $c_{d}$ is in standards referred to as the coefficient forces $c_{f, 0}$ [16]. It takes into account the shape and quality of the body surface and thus affects the size of the resistance force on object.

In the case of a solved smokestack, the unevenness of the surface (roughness) is given by the height of the sheet metal. However, according to the standard, the specific shape of the surface of the flow over a cylinder cannot be distinguished. Due to the ratio of the smokestack diameter and a wave height of the shaped metal sheet, it can be assumed that the shape of the wave can affect the resulting drag coefficient.

The aim of the article is to compare the standard calculation of the drag coefficient $c_{d}$ with numerical modeling with a real geometry, where the grid is designed to respect the shape of the waves, and also with the simplified geometry of the smooth cylinder, where the influence of the rough surface is expressed by the equivalent aerodynamic roughness with using the modified wall function in ANSYS Fluent [14, 15.

\section{Near-Wall Modeling in the Critical Area}

The study deals with the task of the flow over a circular smokestack, when the air flow rate is defined with the Reynolds number $R e=2.4 \cdot 10^{6}$ which means, that the 


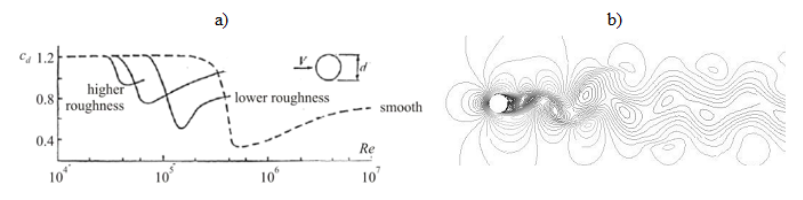

Fig. 1: a) $c_{d}$ coefficient - Re number diagram 14, b) eddy structures behind the cylinder 14

fluid flow problem belongs to the critical area and fully advanced turbulence can be assumed in the boundary layer around the cylinder wall [9]. The effect of the load on the cylinder is expressed by the drag coefficient $c_{d}$. This coefficient changes with the Reynolds number and it is also influenced by the roughness of the surface. This dependency is schematically shown in Fig. 1 1 ). It is clear from the figure how the position of the minimum of a drag coefficient is moved with respect to the surface roughness.

In general, turbulent flows are significantly affected by the presence of wall. The near-wall region can be subdivided into three layers. The innermost layer is called the viscous sublayer, buffer layer follows, the outer layer is called the fully-turbulent layer and turbulence plays there a major role. Toward the outer part of the near-wall region the turbulence is rapidly enlarged by the production of turbulence kinetic energy due to the large gradients in mean velocity. Therefore, accurate representation of the flow in the near-wall region determines successful predictions of wall-bounded turbulent flows [17]. Fig. 10) is for illustrative purposes only and it shows the formation of eddies behind the flow around a cylinder at high Re numbers [14.

Two basic approaches can be used in modeling the near-wall region: using wall functions and near-wall modeling approach [17], see the following paragraphs.

\subsection{Wall Functions}

In the approach of using wall functions for modeling the near-wall region model, the viscosity - affected inner region (viscous sublayer and buffer layer) is not resolved. With these laws it is possible to express the mean velocity parallel to the wall and turbulence quantities outside the viscous sublayer in terms of the distance to the wall. The wall functions are semi empirical formulas and they are used to bridge the viscosity - affected region between the wall and the fully-turbulent region. The use of wall functions obviates the need to modify the turbulence models to account for the presence of the wall [17. The functions are applied in the first cell at the wall, which bridge the distance between the wall and the cell near the wall. Bridging of inner layers reduces the mesh size requirements of the near-wall region and it gives a sufficiently precise solution when dealing with turbulence at the fully-turbulent layer. The use of wall function is therefore suitable for calculations where we are more interested in the center of the system than at the wall. These functions express the dependence of velocity on the distance from the wall, most often in the form dimensionless velocity to the dimensionless distance.

Fluid flows in atmospheric boundary layers over a rough terrain affects the drag coefficient (resistance). It can be possible to include the wall roughness effects through the law-of-the--wall modified for roughness 14. There can be used the simplified geometry and the effect of the shaped casing can be expressed by the so-called equivalent aerodynamic roughness.

\subsection{Near-Wall Modeling Approach}

In another approach, the turbulence models are modified to enable the viscosity-affected region to be resolved with a mesh all the way to the wall, including the viscous sublayer. This approach is particularly useful where wall flow is the most important or recommended for turbulent models for a lower Reynolds number [17]. In this task the influence of the rough casing (the shaped sheathing of the smokestack) can affect the resulting drag coefficient, so a model including near-wall modeling has also been chosen for comparison with the wall functions approach. Detailed modeling of ineer layers means that the radial height of the mesh cells at the wall is sufficiently reduced during crosslinking. With the fineness of the mesh, a detailed description of the flow over the wall including the viscous sublayer is obtained. Since the fluid behavior of the wall is often predictive, it is usually the more effective using the wall functions instead of fine mesh in the case of near-wall modeling.

\section{Task Description}

The study presents the possibility to solve the effects of loads on corrugated sheet metal casing of the smokestack with a numerical solution in ANSYS Fluent software. A numerical SST k-omega model was chosen for the solution, because it is suitable for modeling of flow in near-wall region. Length of computational area $L=140 \mathrm{~m}$, diameter of the smokestack $D=3.6 \mathrm{~m}$. The height of the wave of the shaped sheet is $18 \mathrm{~mm}$. Fig. 2 shows the dimensions of the model and the location of the flow over the cylinder in the computational area. Other parameters of the solved task are: velocity $v=10 \mathrm{~m} / \mathrm{s}$, turbulence intensity $i=10 \%$ and kinematic viscosity $=1.5 \cdot 10^{-5} \mathrm{~m}^{2} / \mathrm{s}$. 


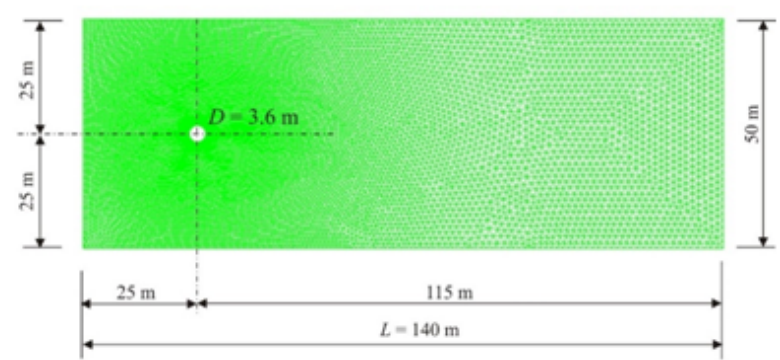

Fig. 2: Computational area of the solved task

Numerical tasks are solved by two approaches. One task solves the effects of load on the simplified model, when the geometry of the chimney is formed by a smooth sheet and the influence of the rough casing is expressed in the calculation by the so-called equivalent aerodynamic roughness. In the second task, the geometry of the model is made up of the real shape of the casing by two different grids, a tetrahedral and a hexahedral grid. All models are in 2D. Figure 3 shows details of the meshes of all the models being solved and they are described in the following paragraphs.

\subsection{Simplified Geometry with the Use of Equivalent Aerodynamic Roughness}

In this option of the calculations, the real shape of the sheathing (corrugated sheet) was replaced with a smooth cross-section of the circular cylinder. The effect of the shaped casing is given by the physical roughness height $K S$ and the roughness constant $C_{S}$, which are used in the calculation for the boundary conditions and which define the aerodynamic roughness equivalent to the actual roughness of the flow around a body [14, [15]. Physical roughness height $K_{S}=18 \mathrm{~mm}$ (height of a wave of the sheet metal) and roughness constant $C_{S}=0.5$. Roughness constant $C_{S}$ depends on the type of the roughness, in the Fluent Manual [17] it is recommended value of 0.5 with a range of $0.5-1.1$. The mesh is formed by a mix of the hexahedral grid and tetrahedral, see Fig. 3a). When creating the mesh, the size of the first cell on the wall must be at least twice as large as the roughness of the sheathing (here the height of the sheet metal) [17]. The hexahedral grid forms the inner layers of the boundary layer to ensure that the wall function works properly. The tetrahedral grid continues further in the computational area.

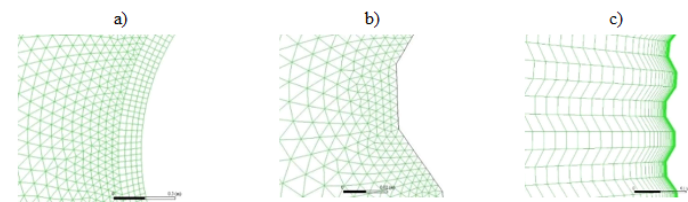

Fig. 3: Meshing of solved models:

a) simplified geometry - hexa/tetra mesh,

b) real geometry - tetrahedral mesh,

c) real geometry - hexahedral mesh

\subsection{Real Geometry of the Cylinder Casing-Tetrahedral Meshing}

In the other two variants, the calculation of the flow over the cylinder with the real sheet metal casing is performed.

The first model has a tetrahedral grid and it is also solved using the wall function in ANSYS Fluent software, see figure Fig. 3b). Unlike the previous option (simplified geometry and aerodynamic roughness), the roughness of the sheathing is given by the real geometry of the casing. Fig. 2 shows a computational area created by a tetrahedral meshing and it is possible to notice the fine mesh around the wall and the enlarging cells in the computational area, which is easily realized in $2 \mathrm{D}$ using a tetrahedral grid.

\subsection{Real Geometry of the Cylinder Casing-Hexahedral Meshing}

In this variant, the calculation is also performed on the real geometry of the smokestack casing modeled by the hexahedral grid, see Fig. 3p) and the task is solved in ANSYS Fluent software as a task of nearwall modeling approach. The created grid respects the shape of the casing and thus the real geometry and it was created to prepare calculations in 3D in future research.

\section{Results of the Solution}

The results of drag coefficients obtained from all variants of the numerical models are compared to the standard calculation and described in the following paragraphs.

\subsection{Drag Coefficient from Numerical Modeling}

The time record of the solved drag coefficient obtained from all three variants of numerical simulations is shown in the graphs in Fig. 4. The average value of 

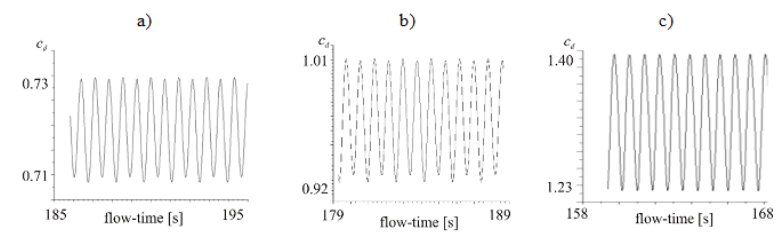

Fig. 4: Drag coefficient obtained from numerical simulations:

a) simplified geometry,

b) real geometry-tetrahedral mesh,

c) real geometry- hexahedral mesh

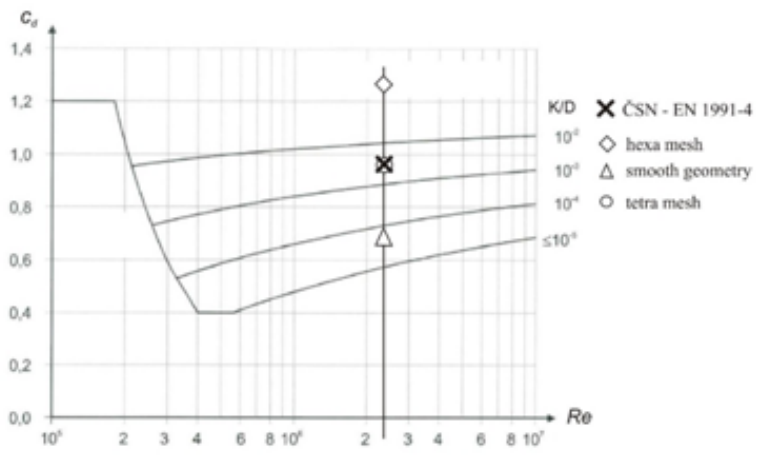

Fig. 5: The value of the aerodynamic drag coefficient-scheme from EN 1991-1-4 [17

drag coefficient $c_{d}$ in the task with simplified geometry $c_{d}=0.72$, in the variant based on a real geometry with the tetrahedral mesh $c_{d}=0.96$ and in a real geometry task with hexahedral mesh $c_{d}=1.31$.

\subsection{Drag Coefficient According to the Standard}

Determining of the drag coefficient according to the standard depends on the size of the Reynolds number $\left(R e=2.4 \cdot 10^{6}\right)$ and on the ratio of the roughness $K$ and the diameter $D$ of the cylinder $(K / D=0.018 / 3.6=5$. $\left.10^{-3}\right)$. Drag coefficient is determined according to the standard graph from EN 1991-1-4. Scheme of the graph can be seen on Fig. 5 and drag coefficient according to standard is $c_{d}=0.95$.

The graph on Fig. 5 also shows the average resultant of the drag coefficients obtained by the numerical solutions of all three mentioned variants both on the simplified and the real geometry of the jacketed smokestack.

\section{Conclusion}

The study presented the possibility to solve the effects of load on smokestack with the rough casing by numerical solution in 2D in ANSYS Fluent software, which was compared with the standard calculation. Numer- ical tasks were solved by two approaches. The first solution of the task was achieved on a simplified geometry, when the casing was made of a smooth sheet and the influence of the rough casing was expressed in the calculation by the so-called equivalent aerodynamic roughness. In the second task, the geometry of the smokestack was made up of a real shape of casing using two models, one of which was created by a tetrahedral grid, and the second one by a hexahedral grid. As it has been assumed, numerical solutions have achieved different drag coefficient values.

The result obtained by calculating with the real geometry modeled by the tetrahedral mesh almost matches with the standard results and it can be evaluated as the best one. However, the results obtained in the two other variants differ from the standard calculation. In the case of the real hexa-shaped geometry task, it is problematic to create a grid just close to the wall (the shaped metal sheet), by adjusting the ratio of sides in the grid may be achieved a more relevant result.

In the case of simplified smooth geometry using the aerodynamic roughness it is a relatively complex problem of correct setting of the physical roughness height $K S$, because in the numerical solution the coefficient $K S$ was given only by the height of the wave and the shape of the chimney casing could not be taken into account. This issue will be the subject of further research.

\section{Acknowledgment}

The paper has been supported by the project of "Conceptual development of science and research activities 2018" on the Faculty of Civil Engineering, VSB-TU Ostrava and by the Ministry of Education, Youth and Sports from the Large Infrastructures for Research, Experimental Development and Innovations project "IT4Innovations National Supercomputing Center-LM2015070".

\section{References}

[1] MERRICK, R. and G. BITSUAMLAK. Control of flow around a circular cylinder by the use of surface roughness: A computational and experimental approach. In: Proceeding of the 4th International Conference Advances on Wind and Structures (AWAS08). Jeju, Korea, 2008.

[2] DOBES, J. and M. KOZUBKOVA. The influence of numerical models on determining the drag coefficient. In: Proceeding of the International Conference on Experimental Fluid Mechanics. Kutna 
Hora, Czech Republic, 2013. EPJ Web of Conferences.

[3] ROSHKO, A. Experiments on the flow past a circular cylinder at very high Reynolds number. Journal of Fluid Mechanics. 2006, X. Nr. 1, pp. 345-356. ISSN 0022-1120.

[4] HUBOVA, O., KONECNA, L. and P. LOBOTKA. Influence of Walls and Ceiling on a Wind Flow in BLWT Tunnel. Applied Mechanics and Materials. 2014, vol. 617, pp. 257-262.

[5] CATAlanO, P., WANG, M. and G. IACCARINO. Numerical simulation of the flow around a circular cylinder at high Reynolds numbers. Journal of Heat and Fluid Flow. 2003, vol. 24, pp. 463-469.

[6] BREUER, M. A challenging test case for large eddy simulation: high Reynolds number circular cylinder flow. Journal of Heat and Fluid Flow. 2000, vol. 21, pp. 648-654.

[7] LAKEHAL, D. Computation of turbulent shear flows over rough-walled circular cylinders. Journal of Wind Engineering and Industrial Aerodynamics. 1999, LXX. Nr. 1-2, pp. 47-68. ISSN 01676105 .

[8] XU, CH., CHEN, L. and X. LU. Large-Eddy and Detached-Eddy Simulations of the separated flow around a circular cylinder. Journal of Hydrodynamic. 2007, IXX, Nr. 5, pp. 559-563. ISSN 10016058.

[9] SQUIRES, K.D., KRISHNAN, V. and J.R. FORSYTHE. Prediction of the flow a circular cylinder at high Reynolds number using detachededdy simulation. Journal of Wind Engineering and Industrial Aerodynamics. 2008, XCVI, pp. 1528-1536. ISSN 0167-6105.

[10] NiSHINO, T., ROBERTS, G.T. and X. ZHANG. Unsteady RANS and detached-eddy simulations of flow around a circular cylinder in ground effect. Journal of Fluids and Structures. 2008, XXIV, Nr. 1, pp. 18-33, ISSN 0889-9746.

[11] SHUR, M.L. Unsteady A hybrid RANS-LES approach with delayed-DES and wall-modelled LES capabilities. Journal of Heat and Fluid Flow. 2008, XXIX, pp. 1638-1649. ISSN 0142-727X.

[12] WANG, M., CATAlANO, P. and G. IACCARINO. Prediction of high Reynolds number flow over acircular cylinder using LES with wall modelling. Center for Turbulence Research. pp. 45-50, Stanford University, 2001.
[13] LEOVEANU, I.S. [et al.]. Computer fluid dynamics application for establish the wind loading on the surfaces of tall buildings. In:Proceedings of the 13th WSEAS international conference on Automatic control, modelling and simulation. Spain, 2011, WSEAS, pp. 433-438. ISBN 978-1-61804004-6.

[14] MICHALCOVA, V. and L. LAUSOVA. Numerical approach to determination of equivalent aerodynamic roughness of Industrial chimneys. Computers and Structures. 2018, vol. 207, pp. 187-193. ISSN 0045-7949. DOI: $10.1016 /$ j.compstruc.2017.03.013.

[15] MICHALCOVA, V. and L. LAUSOVA. Numerical Calculation of Aerodynamic Roughness of Chimney Jacketed with Corrugated Sheets. Transactions of the VSB-Technical University of Ostrava, Civil Engineering Series. 2016, vol. 16, iss. 1, pp. 33-39, ISSN (Online) 1804-4824, DOI: $10.1515 /$ tvsb-2016-0006.

[16] CSN-EN 1991-4. Eurocode 1: Actions on structures - Part 1-4: General actions Wind actions. Praha: Ceský normalizacni institut, 2007.

[17] Ansys, Inc. ANSYS FLUENT 17.0 - Theory Guide. pp. 826. 2016. 